Brandes, C. M., Herzhoff, K., Smack, A. J., \& Tackett, J. L. (in press). The $p$ factor and the $n$ factor: Associations between the general factors of psychopathology and neuroticism in children. Clinical Psychological Science.

The $p$ Factor and the $n$ Factor:

Associations Between the General Factors of Psychopathology and Neuroticism in Children

Cassandra M. Brandes

Kathrin Herzhoff

Avanté J. Smack

Jennifer L. Tackett

Northwestern University

Author Note

Correspondence concerning this article should be addressed to Cassandra M. Brandes, Department of Psychology, Northwestern University, Evanston, IL 60208.

Contact: cbrandes@u.northwestern.edu 


\begin{abstract}
Research across age groups has consistently indicated that psychopathology has a general factor structure, such that there is a broad latent dimension (or $p$ factor) capturing variance common to all mental disorders, as well as specific internalizing and externalizing factors. This research has found that the $p$ factor overlaps substantially with trait negative emotionality (or neuroticism). However, less is known about the psychological substance of the specific factors of the general psychopathology model, or how lower-order facets of neuroticism may relate to each psychopathology factor. We investigated the structure of neuroticism and psychopathology, as well as associations between these domains in a sample of 695 pre-adolescent children using multi-method assessments. We found that both psychopathology and neuroticism may be wellcharacterized by bifactor models, and that there was substantial overlap between psychopathology $(p)$ and neuroticism $(n)$ general factors, as well as between specific factors (Internalizing with Fear, Externalizing with Irritability). Keywords: comorbidity, neuroticism, developmental psychopathology, multimethod assessment
\end{abstract}


The $p$ Factor and the $n$ Factor:

Associations Between the General Factor of Psychopathology and Neuroticism in Children A clear and accurate understanding of psychopathology structure is critical for informing classification and assessment. Historically, traditional psychiatric nosologies such as the Diagnostic and Statistical Manual of Mental Disorders (DSM) have defined disorders based on clinical consensus, and as discrete phenomena. However, empirical study of the structure of these disorders has shown that there is substantial covariation, or comorbidity between clinical problems (Krueger \& Markon, 2006). A wealth of research now suggests that there is a need for a comprehensive framework for studying the classification, etiology, and treatment of psychopathology that incorporates both broad and specific dimensional factors of psychological functioning (i.e. the Hierarchical Taxonomy of Psychopathology, or HiTOP; Kotov et al., 2017). Recent investigations of overall psychopathology structure model general and specific disorder factors at the broadest levels of analysis (i.e., in a bifactor model; Caspi et al., 2014; Lahey et al., 2012; Tackett, Lahey, et al., 2013), yet these models are in need of further replication and extension across different populations. In addition, the substantive, or psychological, nature of these bifactor models has only recently been interrogated. The purposes of the present study were to: 1) examine these psychopathology bifactor models in a large sample of pre-adolescent children, 2) to extend structural models of trait neuroticism (the personality trait implicated in psychopathology bifactor models to date; Caspi et al., 2014; Olino, Dougherty, Bufferd, Carlson, \& Klein, 2014; Tackett, Lahey, et al., 2013), and 3) to explore overarching associations between general and specific factors of psychopathology and trait neuroticism.

\section{The Internalizing-Externalizing Model}


Early examination of the covariation between psychiatric syndromes was done through factor analytic study of children's behavior problems (Achenbach, 1966). The resulting twofactor model of psychopathology includes internalizing (e.g. social anxiety, major depression) and externalizing problems (e.g. substance abuse disorders, conduct disorder). This model has proven robust across many samples, age groups, and clinical/nonclinical status, and it currently dominates research in empirically based psychopathology assessment (Achenbach \& Edelbrock, 1978; Forbes, Tackett, Markon, \& Krueger, 2016; Krueger \& Markon, 2006).

The two-factor model of psychopathology has been successful both due to its extensive empirical support and its clinical utility. These two factors are assumed to reflect latent, dimensional variables, in contrast to the categorical DSM approach. This two factor structure is recovered in factor analyses of both empirically derived dimensional instruments such as the Child Behavior Checklist (CBCL; Achenbach \& Edelbrock, 1978), as well as DSM-based diagnostic measures (Krueger \& Markon, 2006). Further, scores on these latent factors of psychopathology have stronger predictive power for future psychopathology than do discrete diagnoses (Kim \& Eaton, 2015). Predictive validity is an important criterion for usefulness in both clinical and academic domains, and given the relative instability of categorical psychopathology diagnoses (Markon, Chmielewski, \& Miller, 2011), this is an important point of improvement over historical psychopathology models.

One of the more robust and replicable features of the internalizing-externalizing twofactor model is the substantial overlap been the internalizing and externalizing factors. The metaanalytic estimate of the correlation between internalizing and externalizing factors is approximately .50 (Krueger \& Markon, 2006). Further, some individual syndromes (e.g. depression) tend to be substantially comorbid with disorders across the internalizing and 
externalizing spectra (e.g. with both anxiety and substance abuse). That is, some disorders may not be sufficiently discriminating between internalizing and externalizing factors, and may instead be more direct indicators of a broad predisposition to psychopathology (Caspi et al., 2014; Lahey et al., 2012; Tackett, Lahey, et al., 2013). This covariation between spectra has implications for the development of psychiatric classification systems, as it indicates that there is a need to identify and explore higher levels of dysfunction (e.g. the "superfactors" of HiTOP; Kotov et al., 2017).

\section{The $p$ Factor}

Given the substantial association between internalizing and externalizing symptoms, there has been a desire to quantify the extent to which all clinical problems covary (Caspi et al., 2014; Lahey et al., 2012; Martel et al., 2016). In the "bifactor" model, there is a general factor $p$ (which parallels $g$ in the intelligence literature), as well as separable internalizing- and externalizing-specific factors. While $p$ reflects systematic covariation across the entire gamut of psychiatric problems, the specific factors reflect unique variance among internalizing and externalizing symptoms, after accounting for general variance in $p$. From a psychological perspective, $p$ and the specific factors may reflect dispositional features or vulnerabilities that contribute to the expression or development of particular types of disorders.

While both correlated factors and bifactor models show good overall fit, applying a bifactor model to psychopathology offers the unique advantage of differentiating common features that predict all forms of psychopathology from distinct features that predict specific forms of dysfunction. Multiple studies have demonstrated that the general factor of psychopathology correlates with known predictors of all-form psychopathology, including intelligence (Caspi et al., 2014), executive functions (Martel et al., 2016), and chronic stress 
(Snyder, Young, \& Hankin, 2017), while specific factors show weak to null relationships with these external criteria. In contrast, general predictors such as intelligence correlate nonspecifically with multiple subfactors or spectra within hierarchical psychopathology models (Caspi et al., 2014), indicating that where researchers wish to separate transdiagnostic from specific features or vulnerabilities for psychopathology, the bifactor model may be a more useful model to employ. The general factor model of psychopathology has been investigated in studies of adults and (to a lesser extent) children, with consistent support for the reliability of bifactor models of psychopathology in a variety of populations and study designs (Caspi et al., 2014; Laceulle, Vollebergh, \& Ormel, 2015; Lahey et al., 2012; Murray, Eisner, \& Ribeaud, 2016; Tackett, Lahey, et al., 2013).

Given this emerging consensus in the literature, researchers have recently turned to a more substantive question: what is the $p$ factor? Studies attempting to delineate the nature of psychopathology have frequently found both structural and empirical parallels between personality and psychopathology, with some indication that traits and mental illness may have shared measurement (Krueger \& Markon, 2006; Krueger \& Tackett, 2003; Tackett, 2006). In considering those dispositional factors that may be related to general psychopathology variance, one strong candidate is trait neuroticism, or negative emotionality (Barlow, Sauer-Zavala, Carl, Bullis, \& Ellard, 2014; Kotov, Gamez, Schmidt, \& Watson, 2010; Tackett, Lahey, et al., 2013), which we now turn to.

\section{Neuroticism and Psychopathology}

Neuroticism is a primary personality trait reflecting a tendency to experience negative emotions, and it is robustly associated with many forms of psychopathology (Barlow et al., 2014; Griffith et al., 2010; Zinbarg et al., 2016). Neuroticism's association with both internalizing and 
externalizing psychopathology is the most consistent of any trait in the Five Factor Model (Kotov et al., 2010). It is a highly replicable predictor of depression and other internalizing disorders, and this connection has been observed both cross-sectionally and longitudinally (Barlow et al., 2014; Zinbarg et al., 2016). Among externalizing disorders, the connection between neuroticism and substance use disorders has been the most well-documented (e.g. Kotov et al., 2010; Zinbarg et al., 2016). However, trait negative emotionality has also been connected to a variety of other externalizing problems, including aggressive and rule breaking problems in children and adults, as well as comorbid externalizing and internalizing problems (Eisenberg et al., 2009; Tackett \& Lahey, 2017).

The general factor of psychopathology has itself been examined in relation to trait negative affectivity, and specifically in children. In a study by Tackett and colleagues (2013), $p$ and its specific factors were examined in relation to trait negative emotionality on both phenotypic and genotypic levels. This study found that negative emotionality strongly and specifically (i.e., discriminant associations were found for other dispositional traits) overlapped with the $p$ factor at both phenotypic $(r=.58)$ and genetic $(r=.71)$ levels. However, this study did not use a broadband measure of trait neuroticism, but rather, a measure of negative emotionality that was designed to assess vulnerability for externalizing problems (the Child and Adolescent Dispositions Scale; Lahey et al., 2008). Further research has replicated this association between $p$ and neuroticism/negative affectivity in adults (Caspi et al., 2014) and preschool-aged children (Olino et al., 2014) using broader individual difference measures. Despite this, there is a clear need to investigate this association across a broader age range, and across different aspects of negative emotionality. Though preliminary evidence suggests that neuroticism may also be characterized by a bifactor model with general and specific factors (Zinbarg et al., 2016), no 
studies to date have examined how a neuroticism bifactor may be related to the psychopathology bifactor, or how this parallel structure may be informative regarding the psychological content of the general psychopathology model. Further research is needed to examine how inclusion of both general and specific factors of neuroticism may add to our understanding of general and specific factors of psychopathology.

Neuroticism can be reliably measured as early as age 3 (Tackett, 2006, 2011), but it is nonetheless a particularly challenging trait to measure in children. The reliance on informant reports in child personality research may present as especially challenging with neuroticism, which indexes many internal states (Tackett, 2011). Other developmental considerations in the measurement of neuroticism include higher covariation between neuroticism and (low) agreeableness (i.e., antagonism) in youth, relative to adults, which indicates potential developmental differences in trait coverage that may impact associations with other constructs, including psychopathology (Soto \& Tackett, 2015). In temperament models (De Pauw, 2017) as well as factor analytic studies of primary personality traits in children (Tackett et al., 2012), separable factors for neuroticism and agreeableness are often not recovered, leading to questions about the utility of distinguishing these two traits in children. Furthermore, the lower-order structure of child personality traits is poorly defined. There are slight differences between measurement models, but only two facets of child neuroticism have been consistently recovered from bottom-up research: fear and irritability (De Pauw, 2017; Shiner \& Caspi, 2003). There is clearly a need for better coverage of lower-order facets of neuroticism in empirical studies, and attention to structural models of neuroticism in children, particularly given its high relevance for psychopathology. 
Evidence suggests that, despite conceptual overlap between measures of child personality and temperament, the two measurement approaches may capture non-overlapping variance in dispositional traits (Tackett, Kushner, De Fruyt, \& Mervielde, 2013). Across all traits, a combination of temperament and personality measures seems to predict a greater proportion of variance in child psychological problems better than each alone. Further, combined temperament-personality measures may discriminate between forms of psychopathology better than individual measures (De Pauw, Mervielde, \& Van Leeuwen, 2009). Considering neuroticism specifically, personality measures may emphasize aspects of fear and anxiety more so than irritability (e.g., Halverson et al., 2003). The corresponding temperament domain negative affectivity - includes more indicators of anger and irritability, on the other hand (e.g., Simonds \& Rothbart, 2004). A combinatorial approach to measurement of child dispositions using both temperament and personality assessment tools may provide researchers with a more comprehensive framework for the study of child negative emotionality, particularly vis-à-vis external constructs such as psychopathology.

\section{The Present Study}

The goals of the present study were to investigate the structure of both common psychopathology and neuroticism in children, and to gain a better understanding of associations between the two. The present study had four primary aims:

1. We investigated the structure of common psychopathology in children through model comparison in a large sample of children, including both empirically derived and diagnostically based assessments of clinical problems. We hypothesized that a bifactor structure would describe these data better than an two factor (internalizing-externalizing) 
structure, as has been observed in previous research (Caspi et al., 2014; Lahey et al., 2012; Martel et al., 2016; Olino et al., 2014; Tackett, Lahey, et al., 2013).

2. We used the same method to analyze the structure of neuroticism in children, incorporating both personality and temperament measures. We did not have specific hypotheses about whether a bifactor or two factor (fear-irritability) structure would best describe the data, as to the authors' knowledge, this comparison has never before been made in child personality research.

3. We used the best-fitting models for psychopathology and neuroticism to examine associations between individual differences in negative emotionality and common psychopathology. In a two-factor model of psychopathology, we expected that the general neuroticism factor $(n)$ would correlate substantially with both Internalizing and Externalizing factors, and that Fear- and Irritability-specific factors would be substantially correlated with Internalizing and Externalizing factors, respectively. In the model with both psychopathology and neuroticism bifactors, we hypothesized that, of all psychopathology-personality correlations in the model, the general factor of psychopathology $(p)$ would be most strongly related to $n$. Further, we hypothesized that Fear- and Irritability-specific factors would overlap most strongly with Internalizing- and Externalizing-specific factors, respectively. However, relationships between psychopathology specific factors and other factors in the neuroticism model (e.g., Fearspecific and Externalizing-specific) would be markedly lower.

\section{Methods}

\section{Participants}


Participants in this study were taken from two separate longitudinal samples (referred to as Samples $1 \& 2$ ), with no overlapping individuals between them. Sample 1 is originally described in Tackett (2011), and all families from the original sample were included here. Sample 2 is originally described in Tackett, Herzhoff, Smack, Reardon, \& Adam (2017), and all but one family (missing all relevant variables) from the original sample were included here.

Participants were 711 primary caregivers of 695 7-to-13-year-old children $\left(\mathrm{M}_{\text {age }}=9.87\right.$; 48\% male). Parents reported their child's race/ethnicity as 51\% European/White, 16\% AfricanAmerican, 13\% Latino/Latina, 12\% Multi-Racial, 7\% Asian American/Pacific Islander, and $<1 \%$ Other. A 9-point Hollingshead (1975) Occupational Scale was used as a measure of socioeconomic status in Sample 1 (originally reported in Kushner, Tackett, \& Bagby, 2012), with higher ratings on this scale indicating higher status. On this scale, participants in Sample 1 scored 7.02, on average $(\mathrm{SD}=1.66)$. Average household income was used as a measure of socioeconomic status in Sample 2, with categorical options from 1 (under $\$ 20,000$ ) to 10 (over $\$ 100,000)$. On this scale, participants in Sample 2 scored 5.81, on average $(\mathrm{SD}=3.62)$, or approximately $\$ 60,001-70,000$.

Participants in Sample 1 were recruited from a database of families interested in participating in research studies or advertisements posted in the community of a metropolitan area in southern Ontario, Canada. Participants in Sample 2 were recruited from an urban community in the Southwest region of the United States, using directory information from local school districts and flyers posted throughout the community and distributed at events. Inclusion criteria for the studies were fluency in English or Spanish and exclusion criteria were diagnoses of intellectual disability, neurodevelopmental disorders, or psychotic disorders in the child.

\section{Measures}


Child Behavior Checklist (CBCL- 6-18; Achenbach \& Rescorla, 2001). The CBCL is an empirically derived 118-item parent-report measure that assesses eight narrowband problem behavior scales, five of which load onto two broadband Internalizing and Externalizing Problems scales. These five narrowband problem behavior scales include Aggressive Behavior, AnxiousDepressed, Rule-Breaking Behavior, Somatic Complaints, and Withdrawn-Depressed. Caregivers rated each item on a scale from 0 (not true (as far as you know)) to 2 (very true or often true) for the past 6 months. This measure was completed by participants in both samples. Internal consistencies (standardized alphas) were between .67 (Somatic Complaints) and .89 (Aggressive Behavior) for subscales.

Computerized Diagnostic Schedule for Children (C-DISC; Shaffer, Fisher, Lucas, Dulcan, \& Schwab-Stone, 2000). The C-DISC is a structured interview administered to assess children's DSM-IV-based psychopathology symptoms. Items were summed into the following DSM-IV criteria counts: Major Depressive Disorder (MDD), Separation Anxiety Disorder (SAD), Social Phobia (SOPHO), Attention Deficit Hyperactivity Disorder (ADHD, combined), Oppositional Defiant Disorder (ODD), and Conduct Disorder (CD). This measure was completed by participants in both samples. One caregiver and the youth completed the interview, but only parent data was used for these analyses, as youth report personality measures were not collected, and youth ratings on the CDISC introduced substantial interrater variance (for parentyouth report discrepancies, see De Los Reyes \& Kazdin, 2005). All research assistants who administered these interviews underwent a semester-long training prior to data collection (CDISC). Internal consistencies (standardized alphas) ranged from .35 (CD) to .89 (ADHD) for the caregiver report. 


\section{Inventory of Children's Individual Differences- Short (ICID-S; Deal et al., 2007;}

Halverson et al., 2003). The ICID-S is a 50-item shortened parent-report measure that assesses the five higher-order personality traits which are analogous, but not identical, to the Five Factor Model higher-order traits as typically assessed in adults: Neuroticism, Extraversion, Openness to Experience, Agreeableness, and Conscientiousness. The ICID-S also assesses 15 lower-order facets that load onto these higher-order factors. Analyses focused on the higher-order traits Neuroticism and Agreeableness. The corresponding lower-order facets for Neuroticism included Fearful, Shy, and Negative Affect (i.e., irritability). The facets of (reverse) Agreeableness used included Antagonism and Strong-Willed. Caregivers rated each item on a seven-point scale ranging from 1 (much less than the average child) to 7 (much more than the average child). This measure was completed by participants in both samples. Internal consistency (standardized alpha) for Neuroticism was .84 and Agreeableness was .86. Internal consistencies for the facets ranged from .70 (Shy) to .87 (Negative Affect).

\section{Early Adolescent Temperament Questionnaire- Revised (EATQ-R; Ellis \&}

Rothbart, 2001). The EATQ-R is a 62-item questionnaire that assesses temperament in 9-15 year old children. The EATQ-R assesses nine lower-order facets that load onto three higherorder traits: Effortful Control, Surgency, and Negative Affect. Analyses focused on the higherorder trait Negative Affect and its corresponding lower-order facets: Aggression, Frustration and Depressed Mood. Caregivers rated each item on a five-point scale ranging from 1 (almost always untrue of your child) to 5 (almost always true of your child). This measure was completed only by participants in Sample 2. Internal consistency (standardized alpha) for Negative Affect was .88 in Sample 2. Standardized alphas for the facets ranged from .65 (Fear) to .81 (Frustration) in Sample 2 . 


\section{The Temperament in Middle Childhood Questionnaire (TMCQ; Simonds \&}

Rothbart, 2004). The TMCQ is a 157-item questionnaire that assesses temperament in youth. The TMCQ assesses seventeen lower-order facets that load onto three higher-order traits: Effortful Control, Surgency, and Negative Affect. Analyses focused on the higher-order trait Negative Affect and its corresponding lower-order facets: Anger-Frustration, Discomfort, Fear, Sadness, and Soothability. Caregivers rated each item on a five-point scale ranging from 1 (almost always untrue) to 5 (almost always true). This measure was completed only by participants in Sample 1. Internal consistency (standardized alpha) for Negative Affect was .92 in Sample 1. Standardized alphas for the facets ranged from .78 (Discomfort) to .82 (AngerFrustration) in Sample 1.

\section{Procedure}

All measures relevant to our research question, including all primary female caregiverreport psychopathology, personality, and temperament are included here. Only one caregiver completed the CDISC in Sample 1, so where the primary female caregiver CDISC data was missing, the other caregiver's report was used $(\mathrm{N}=42$ fathers). As these data were drawn from two large-scale longitudinal studies which measured many other developmentally relevant constructs (e.g. child life stressors, physical development, parenting style), a complete list of materials cannot be enumerated here. For complete documentation of all measures used in each study, citations for papers using these datasets, measures (as publicly available), and all analytic scripts, see the OSF page for this project at https://osf.io/9fv7z/. Sample 1 questionnaires were completed both at home and at the lab, and computerized interviews were completed at the lab. Sample 2 questionnaires and computerized interviews were completed in the lab (Sample 2). Informed consent was obtained from all participants. Ethics approvals for all procedures were 
obtained from the institutional review boards at the University of Toronto and the University of Houston. Participants were included in the present study if the mother had completed any one of the measures described above, and no other exclusions were made. See relevant publications for additional information about the samples (e.g., Tackett, Herzhoff, Reardon, De Clercq, \& Sharp, 2014; Tackett et al., 2017). Sample 1 caregivers were compensated for their participation with \$40 Canadian for completion of all materials, and children from this sample were given two small gifts. Sample 2 caregivers were compensated with \$75 US gift cards, and children from this sample were given one small gift.

\section{Data Pooling and Measurement Harmonization}

In order to increase the statistical power of our analyses, we pooled data between the two samples in this study. Psychopathology and personality measures were fully overlapping between samples. However, the temperament measures used in each study differed slightly, in that not all indicators overlapped between the measures. Overlapping scales between the measures included Fear, Sadness/Depressed Mood, and Anger-Frustration/Frustration. Nonoverlapping scales between the measures included TMCQ Discomfort, TMCQ Soothability, and EATQ-R Aggression. As such, we harmonized these measures by collapsing samples on only shared scales, and by treating data as missing for the appropriate sample where the scales did not overlap (e.g., Sample 1 completed the TMCQ, so participants from this sample have missing data for EATQ-R Aggression in overall models). See Footnote 1 for details on quality checks for this harmonization method, and the OSF page for this project for results conducted separately by sample.

\section{Results}


Raw data for this project are not openly available, as participating families did not consent to the sharing of individual-level data. However, correlation matrices are available on the OSF page for this project, which allow for full reproduction of the models listed here.

As expected, psychopathology data in these community samples were skewed (see supplemental material on the OSF page for full descriptives), so maximum likelihood estimation with robust standard errors (MLR) was employed for psychopathology and full structural models, while MLE was used for personality models. Eleven percent of data were missing, most of which was accounted for by the nonoverlapping temperament scales. As such, Full Information Maximum Likelihood was used. All analyses were conducted using R statistical software, specifically in the "lavaan", "psych", and "semTools" packages, as well as base R (R Core Team, 2017; Revelle, 2017; Rosseel, 2012; semTools Contributors, 2016). Model fit decisions were made based on Root Mean Square Error of Approximation (RMSEA) values and Standardized Root Mean Square Residual (SRMR). Comparative Fit Index (CFI), Tucker-Lewis Index (TLI) were also used when possible. ${ }^{1}$ CFI and TLI values greater than or equal to .95 were interpreted to reflect good fit, while values greater than .90 were considered to reflect adequate fit. RMSEA values below .06 were interpreted to reflect good fit, while values less than or equal to .10 were taken to reflect adequate fit. SRMR values below .06 were interpreted to reflect adequate fit (Browne \& Cudeck, 1992).

\section{Psychopathology Model Comparison}

\footnotetext{
${ }^{1}$ As our measurement harmonization approach yielded greater missingness for some indicators (those non-overlapping between temperament measures), null models using personality data did not converge. Therefore, relative global fit indices (which reference the null model) are unavailable for these models. As a quality check, we also tested the models without nonoverlapping indicators (less missingness). These models all fit just as well by both absolute and relative global fit indices. Full results are available on the OSF page for this project.
} 
To examine the structure of psychopathology in children, we used Confirmatory Factor Analysis (CFA) to compare several models of psychopathology structure, using data from both the CDISC and CBCL: a two correlated Internalizing (INT) \& Externalizing (EXT) factor model (Model 1), a one-factor model in which all indicators loaded directly onto a general psychopathology ( $p$ ) factor (Model 2), and a bifactor model in which all indicators loaded onto a general $p$ factor and specific INT and EXT factors (Model 3). We also tested a model in which depression variables loaded only onto the $p$ factor in a bifactor model (rather than on INT; Model 4), consistent with some previous studies (Lahey et al., 2012; Tackett, Lahey, et al., 2013). Within each model, we tested both a version (A) without and (B) with an orthogonal method factor (i.e., that did not correlate with substantive factors) to capture assessment mode-specific variance explained by in the CBCL and CDISC. The psychopathology models with method factors are listed in Table 1, including standardized parameter estimates and fit statistics. The comprehensive listing of psychopathology models tested including standardized and unstandardized parameter estimates, confidence intervals, and goodness-of-fit statistics for each can be found on the OSF page for this project (https://osf.io/9fv $7 \mathrm{z} /$ ).

Consistent with previous research (Achenbach, 1966; Lahey et al., 2012; Martel et al., 2016; Tackett, Lahey, et al., 2013), our INT latent factor included CDISC criteria counts for Separation Anxiety Disorder (SAD), Social Phobia (SO), Major Depressive Disorder (MDD), and CBCL Anxious-Depressed (A-D), Withdrawn-Depressed (W-D), and Somatic Complaints (SOM). The EXT latent factor consisted of CDISC criteria counts for Attention Deficit Disorder (combined Hyperactive/Non-Hyperactive types; ADHD), Conduct Disorder (CD), and Oppositional Defiant Disorder (ODD). CBCL Externalizing measures included Rule Breaking 
and Aggression. ${ }^{2}$ This pattern of indicators was used for both models that included INT and EXT factors (Models 1 and 3). Model 4 differed in that it allocated depression variables (W-D and MDD) to the $p$ factor only (not the INT factor).

Psychopathology bifactor Models 3A (depression variables on INT, no method factor) and 4B (depression variables on $p$, no method factor) achieved acceptable fit by most indices. Models 3B and 4A did not converge. Upon inspection of the data, the likely source of model non-convergence appeared to be large differences in variances among indicators with $\mathrm{CD}$ and MDD at the low end $\left(\mathrm{s}^{2}=.33\right.$ and .57 , respectively), and Aggression and ADHD at the high end $\left(s^{2}=25.36\right.$ and 18.75 , respectively). Model 4B had small amounts of negative residual variance on CDISC SAD, though model fit and parameter estimates did not change when this was fixed to 0. The best fitting models had a CBCL method factor, including the two-factor model (Model $1 \mathrm{~B} ; \mathrm{RMSEA}=.07,90 \% \mathrm{CI}[.06, .09], \mathrm{CFI}=.92, \mathrm{TLI}=.89, \mathrm{SRMR}=.05)$ and bifactor model with depression indicators on the general factor alone (Model 4B; RMSEA $=.08,90 \%$ CI $[.07$, $.09], \mathrm{CFI}=.93, \mathrm{TLI}=.87, \mathrm{SRMR}=.04)$. Model 4 (depression indicators on the $p$ factor only) was preferred to Model 3 (depression indicators on the INT and $p$ factors), as WithdrawnDepressed and Major Depressive Disorder had relatively low loadings on the INT factor in Model 3A. Model 1A achieved acceptable fit by most indices (RMSEA $=.08,90 \%$ CI [.06, .09], $\mathrm{CFI}=.92, \mathrm{TLI}=.89, \mathrm{SRMR}=.05)$. Models $1 \mathrm{~A}, 2 \mathrm{~A}$, and $2 \mathrm{~B}($ the two-factor model without a

\footnotetext{
${ }^{2}$ Although CBCL Attention Problems was not allocated to the Externalizing Problems scale in the original CBCL scoring, we included it in preliminary psychopathology models, following prior models of the general factor (e.g. Laceulle et al., 2014). As this caused models not to converge, it was excluded from further analyses. CDISC ADHD was retained, as the pattern of factor loadings and model fit statistics were similar with and without it. Therefore, to increase the reliability of the Externalizing factor, we included ADHD in further analyses.
} 
method factor and both one-factor models) did not achieve acceptable fit by most indices

$(\mathrm{RMSEA} \geq .09, \mathrm{CFI}<.90, \mathrm{TLI}<.90, \mathrm{SRMR} \geq .07)$.

Though the bifactor model with depression variables loading only on $p$ (Model 4B) had overall good fit and an interpretable solution, Separation Anxiety Disorder, Major Depressive Disorder, and Social Phobia had low loadings on the $p$ factor. While unexpected, these results are consistent with prior findings that clusters of psychopathology differ in the extent to which they reflect general versus specific factor variance (e.g., Olino et al., 2014). These results are further interpreted in the Discussion.

\section{Neuroticism Model Comparison}

To examine the structure of neuroticism in children, we conducted CFA to compare several models of neuroticism using both the ICID and TMCQ/EATQ: a two correlated FearIrritability factor model (Model 5), a one-factor model in which all indicators loaded onto a general neuroticism $(n)$ factor (Model 6), and a bifactor model in which all indicators loaded onto a general $n$ factor, as well as specific Fear and Irritability factors (Model 7). Paralleling the psychopathology models, we also tested a model in which Sadness was allocated to the $n$ factor only (Model 8). ${ }^{3}$ As with the psychopathology data, within each model, we tested both a version without (A) and without (B) an orthogonal method factor to capture assessment mode-specific variance in the ICID and TMCQ/EATQ. The neuroticism models with method factors are listed

\footnotetext{
${ }^{3}$ We tested these models i) with neuroticism and negative affectivity facets alone, and ii) including ICID Agreeableness facets "Strong Willed" and "Antagonism", due to the evidence that neuroticism and (dis)agreeableness are less differentiable in youth (Tackett et al., 2012). As the overlap between higher-order $\mathrm{N}$ and A was substantial when the structural model was fitted and general factors allowed to correlate $(r>.70)$, here we only discuss the combined neuroticism/agreeableness models (henceforth referred to simply as neuroticism models). The complete set of results for these analyses can be found on the OSF page for this project (https://osf.io/9fv7z/).
} 
in Table 2, including standardized parameter estimates and fit statistics. The comprehensive listing of neuroticism models tested including standardized and unstandardized parameter estimates, confidence intervals, and goodness-of-fit statistics for each can be found on the OSF page for this project (https://osf.io/9fv7z/).

Drawing from the broader literature on child personality and temperament, our Fear factor included ICID Fearful and Shy, as well as TMCQ/EATQ Fear and Sadness and TMCQ Discomfort. ICID Shy was included in Neuroticism (rather than Extraversion) to adhere to the Halverson et al. (2003) scoring. The Irritability factor consisted of ICID Negative Affect, Strong Will, and Antagonism, TMCQ/EATQ Anger-Frustration, and TMCQ Soothability. This pattern was used for both models that included Fear and Irritability factors (Models 5 and 7). In order to investigate whether neuroticism's structure might mirror the general factor of psychopathology structure found in some prior research and in our psychopathology analyses (Lahey et al., 2012), Model 8 differed in that it allocated Sadness to the general $n$ factor only (not the Fear factor).

Only the bifactor neuroticism models with method factors (Models 7B \& 8B) achieved acceptable fit to the data. The best fitting model had a TMCQ/EATQ method factor (Model 8B; RMSEA $=.04,90 \%$ CI $[.03, .06]$, SRMR $=.03)$. Though the bifactor model with Sadness allocated to the general $n$ and Fear-specific factors achieved similarly good fit (Model 7B; RMSEA $=.04,90 \%$ CI $[.03, .06]$, SRMR $=.03)$, the bifactor model with Sadness allocated only to the $n$ factor (Model 8B) was preferred, as Sadness had a low loading on the Fear-specific factor $(<.10)$. No two or one factor models achieved adequate fit (RMSEA>.10, SRMR $\geq .06)$, and Model 8A (bifactor with Sadness on $n$, no method factor) did not converge. Upon inspection of the data, the lack of convergence in Model 8A appeared likely attributable to the pattern of 
missing data (particularly, nonoverlapping TMCQ and EATQ scales) leading to very low coverage in some cells of the matrix.

\section{Latent Variable Relationships (Structural Models and Factor Score Correlations)}

To examine the relationships between the two dominant models of psychopathology and neuroticism in children, we fit full structural models including the best-fitting neuroticism model (Model 8) and both the two-factor INT-EXT and bifactor psychopathology models (Models 1 and 4). For the two-factor psychopathology SEM, we allowed all personality factors to correlate with both INT and EXT factors, as well as for the INT and EXT factors to correlate (Model 9). For the bifactor psychopathology SEM (Model 10), we evaluated a model with only hypothesized correlations (between the general factors, and each pair of specific factors), as well an exploratory model in which all between-domain latent variables were allowed to correlate. As the latter model resulted in a latent variable covariance matrix that was not positive definite (likely due to some of the same difficulties in model fitting outlined above), we also extracted factor scores via the regression method (Rosseel, 2012) to examine exploratory correlations between all psychopathology and neuroticism factors using these measurement models. As with the measurement models, these models were all fit both without (A) and with (B) fully orthogonal method factors (i.e., correlations between the method factors themselves and between method factors and substantive factors were fixed to 0 ).

In the two-factor psychopathology model, we expected that the INT and EXT factors would both correlate substantially with the general $n$ factor, as well as Fear- and Irritabilityspecific factors, respectively. We expected that the bifactor psychopathology model would show more discrimination than the two-factor model, such that relationships between the specific factors INT-Fear and EXT-Irritability would be high, while relationships between conceptually 
dissimilar factors (e.g., INT-Irritability specific factors) would be low. We expected that the specific INT-Fear and EXT-Irritability factor correlations would be higher than the INT-Fear and EXT-Irritability correlations would be in the two-factor SEM, as the specific factors in the bifactor model would isolate specific internalizing and externalizing variance from general psychopathology (vs. the two-factor, in which correlations would index both general and specific variance). As previously stated, we expected that the general factor of psychopathology would correlate strongly with the general $n$ factor. Model 10B (both bifactor measurement models with method factors and restricted latent variable correlations) is illustrated in Figure 1, and standardized parameter estimates and fit statistics for both SEMs can be found in Table 3. The comprehensive listing of structural models tested including standardized and unstandardized parameter estimates, confidence intervals, and goodness-of-fit statistics for each can be found on the OSF page for this project.

All models achieved acceptable fit, though both structural models with method factors (9B and 10B) were superior (see Table 3). Model 9B (two-factor psychopathology and bifactor neuroticism) confirmed our hypotheses that the general factor of neuroticism would be robustly associated with both INT $(r=.76, p<.001)$ and EXT $(r=.49, p<.001)$ factors. Further, INT was also significantly correlated with the Fear specific factor $(r=.40, p<.01)$, and EXT was significantly correlated with the Irritability specific factor $(r=.66, p<.001)$. Correlations between INT and the Irritability-specific factor $(r=-.03, p>.05)$, and EXT and the Fear-specific factor $(r=.10, p>.05)$ were nonsignificant.

Correlations between latent variables in Model 10B (both bifactors) also confirmed the predicted associations between $n$ and $p(r=.81, p<.001)$ and Irritability-specific and EXTspecific $(r=.88, p<.001)$. Fear-specific and INT-specific factors were correlated to a strong 
degree $(r=.69, p=.05)$, but there was greater imprecision in this estimate. Exploratory latent variable correlations and factor score regressions indicated that relationships between conceptually dissimilar factors (e.g., $n$ and INT-specific, Fear-specific and EXT-specific) were null to small in size (see Table 4).

\section{Discussion}

Results from this study suggest that the general factor of psychopathology (" $p$ ") overlaps substantially with a general neuroticism factor (" $n$ ”) in children. Specifically, we found that the structure of both neuroticism and psychopathology could be well described by a general bifactor structure, each with two specific factors. These specific factors captured trait fear and irritability in the neuroticism model and internalizing and externalizing in the psychopathology model. The overlap between the general factors of psychopathology and neuroticism, as well as specific externalizing and irritability factors was strikingly large. Further, these results suggest that specific factors of internalizing psychopathology and fear traits overlap as well, though this estimate was perhaps less reliable than those of general and externalizing-irritability factors, and therefore should be interpreted as preliminary evidence. Contrary to expectations, psychopathology data could be equally well-captured by a two-factor internalizing-externalizing model judging by global model fit statistics. However, our results also showed that a bifactor model has greater specificity in its correlations with various aspects of neuroticism. Specifically, the bifactor psychopathology model better separated variance due to a general predisposition toward trait negative emotionality from specific variance in fearfulness and irritability/anger. Overall, these findings suggest that the overarching content of a bifactor model of psychopathology is largely overlapping with the overarching content of a bifactor model of neuroticism in children. 
These results replicated several prior findings on the general factor of psychopathology. Our analyses generally found that a bifactor model fit psychopathology data, as in prior research on this topic (Caspi et al., 2014; Laceulle et al., 2015; Lahey et al., 2012; Martel et al., 2016; Tackett, Lahey, et al., 2013). We further replicated findings that depression variables are stronger indicators of general factor variance alone, rather than both general and internalizing-specific variance (Lahey et al., 2012; Tackett, Lahey, et al., 2013). Our analyses of the structure of neuroticism replicated prior findings that neuroticism overlaps heavily with (dis)agreeableness in children to form a unified N/A factor, as (dis)agreeableness facets fit better with the neuroticism model than on a separate agreeableness factor (see Footnote 3; Tackett et al., 2012). We also replicated and extended findings that trait negative affectivity correlates highly with the general factor of psychopathology (Tackett, Lahey, et al., 2013).

In a novel contribution, these results indicate that in addition to psychopathology, neuroticism may be modeled in a bifactor structure in children. Specifically, all forms of trait negative emotionality (both those expressed internally and externally) covary substantially, though there are subordinate clusters of behavior reflecting two major dimensions of negative emotionality: fear and irritability (De Pauw, 2017; Shiner \& Caspi, 2003). Further, the general factor of neuroticism in this model correlated substantially $(r=.81)$ with the general factor of psychopathology. This suggests that the general psychopathology factor is picking up on much of the same trait content as various measures of neuroticism do. The content of the specific Irritability factor also showed substantial overlap with the Externalizing specific psychopathology factor. Fear- and Internalizing-specific factors also overlapped substantially, but there were some indications that this relationship was less precise, possibly due to the estimation of the Internalizing specific factor itself. This observation is concurred by its lower 
reliability $(\alpha=.56)$ in comparison to EXT-specific $(\alpha=.70)$ and $p(\alpha=.75)$. This is not an uncommon finding in studies of internalizing psychopathology in this age group, particularly using parent reports, as externalizing behavior is generally more observable than internalizing problems (Achenbach, McConaughy, \& Howell, 1987; De Los Reyes \& Kazdin, 2005). These results indicate that relationships between INT- and Fear-specific factors should be treated as tentative and require further replication. Overall, these results suggest that the residual variance in externalizing psychopathology beyond the $p$ factor is likely associated with irritability-related specific facets of neuroticism, with some indication that the same may be true for internalizing psychopathology and fear-specific facets.

There was a high degree of specificity in the correlations between the latent factors in the bifactor $p$ and $n$ models, as well. We found that relationships between conceptually dissimilar factors (e.g., INT-specific and Irritability-specific) were small to null, while correlations between general $p$ and $n$, Fear- and INT-specific, and Irritability- and EXT-specific factors were large. In contrast, when we examined the INT-EXT model in relation to neuroticism, we found that EXT and INT each overlapped with both general $n$ and specific Irritability and Fear factors, respectively. These relationships indicate that these two factors contain variance attributable to both a general liability to experience psychopathology (negative emotionality) and specific vulnerability to externalizing (irritability traits) and possibly internalizing (fear traits). Therefore, the two-factor model — despite being a viable alternative model for the description of psychopathology structure in our study - may have more limited power to predict specific forms of psychopathology in comparison to a bifactor model, as general risk is not differentiated from specific risk within this measurement approach. These findings indicate that the structure and content of the bifactor model of psychopathology is overlapping with the structure and content of 
a bifactor model of neuroticism, demonstrating how a bifactor model of psychopathology may be optimally employed as a tool to test questions about transdiagnostic features or risk factors for psychopathology.

We argue here for the use of a bifactor model of psychopathology not simply due to good global fit, but due to its usefulness in partitioning general and specific variance in psychopathology in order to examine common and distinct vulnerability factors in tandem. Arguments have been raised that data are biased toward bifactor models, such that they will generally fit better than alternative (e.g., correlated factor) models due to their greater parameterization (Morgan, Hodge, Wells, \& Watkins, 2015). While this is sometimes true, we point to the need to examine these models on the basis of their construct validity, rather than just statistical fit (Cronbach \& Meehl, 1955). In the domain of discriminant validity, our results favor the bifactor model. Specifically, our analyses showed that, in a two-factor psychopathology model, INT and EXT factors captured both general vulnerabilities (i.e., the content overlapping with general $n$ ), and specific vulnerabilities (i.e., fearfulness or irritability). However, the bifactor model of psychopathology overlapped much more specifically with neuroticism, such that we found amplified correlations between Fear-specific and INT-specific factors (relative to Fearspecific and INT) and Irritability-specific and EXT-specific factors (relative to Irritabilityspecific and EXT). While our results and prior research (e.g., Caspi et al., 2014; Kotov et al., 2017; Krueger \& Markon, 2006) demonstrate that both bifactor and correlated (or hierarchical) factor models are likely valid descriptors of the structure of psychopathology, as we show, discriminating general from specific variance allows us to partition what makes various forms of psychopathology alike from what makes them distinct: an important feature of models used to examine the causes and expression of psychopathology going forward. 
Taken together, these results suggest that the general psychopathology factor is highly overlapping with the psychological content captured by neuroticism, insofar as it captures commonalities in internalizing and externalizing psychopathology. This finding replicates and extends prior research finding connections between these two constructs (Caspi et al., 2014; Olino et al., 2014; Tackett, Lahey, et al., 2013). One interpretation of these findings is that the vulnerability factors underlying trait negative emotionality may be largely the same as those underlying a general predisposition toward psychopathology (i.e. a spectrum model of psychopathology; Tackett, 2006). This interpretation is corroborated by previous research showing that measures of neuroticism and psychopathology capture a unitary construct, with personality measures capturing less severe levels along this joint dimension (De Bolle, Beyers, De Clercq, \& De Fruyt, 2012). Prior research supporting this interpretation has also indicated genetic overlap between neuroticism and externalizing psychopathology, internalizing psychopathology, and a general psychopathology factor (Gjone \& Stevenson, 1997; Hettema, Neale, Myers, Prescott, \& Kendler, 2006; Krueger et al., 2002; Schmitz et al., 1999; Tackett, Lahey, et al., 2013). There is also evidence for shared psychobiological factors (e.g. amygdala dysregulation, hypothalamic-pituitary-adrenal axis dysfunction) between neuroticism and multiple common forms of psychopathology including anxiety, depression, and substance abuse (see Ormel et al., 2013 for review). Collectively, these findings suggest that, at the least, neuroticism and the broad disposition toward psychopathology co-occur at multiple levels of analysis (genetic, neural, endocrine, and phenotypic).

Despite substantial evidence for the spectrum model, however, prior research does not uniformly support this interpretation of neuroticism-psychopathology overlap. Some research indicates that dimensions of psychopathology outside of common mental disorders — including 
personality disorders and thought disorders - in particular may not conform well to a spectrum model (Suzuki, Samuel, Pahlen, \& Krueger, 2015; Walton, Roberts, Krueger, Blonigen, \& Hicks, 2008). There is also evidence for the validity of multiple other causal models to explain the relationship between psychopathology and personality, including those in which traits predispose individuals to psychopathology (the vulnerability model), affect symptom manifestation or severity (the pathoplasty model), or are changed by the experience of psychopathology (the scar model; Ormel, Jeronimus, et al., 2013; Tackett, 2006). Thus, these results must be considered in the context of prior research on shared etiological factors between psychopathology and neuroticism, and research that incorporates a wider variety of indicators of psychopathology as well as more causally informative designs must be done to further test the interpretation that psychopathology and neuroticism capture different points on a joint spectrum.

An alternative interpretation of the present results is that common item content between neuroticism and psychopathology measures accounts for the observed overlap between these two constructs, rendering correlations tautological (Nicholls, Licht, \& Pearl, 1982). Our data and prior studies offer evidence against this interpretation, however. First, among the measures in our study, few items were explicitly or implicitly shared between trait and psychopathology measures (e.g., between the CBCL and personality/temperament measures, no items were shared verbatim, and only $12 \%$ of CBCL items closely matched an individual difference item). Second, prior studies that have examined correlations between neuroticism and psychopathology have observed similar results even after removing overlapping items (e.g., Walton, Pantoja, \& McDermut, 2018). Third, item content overlap between psychopathology and neuroticism measures is a necessary observation within the spectrum model of psychopathology (Ormel, Jeronimus, et al., 2013). Further, the generation of multiple items with similar (though not 
verbatim) wording is a primary strategy for creating unidimensional psychological measures

(Clark \& Watson, 1995). As such, even the small amount of item overlap in our study would not negate the interpretation that neuroticism and general psychopathology measure shared psychological content.

These findings may also point to a better conceptualization of various disorders at the trait level. Prior research has found that, while there is comorbidity between all forms of psychopathology, clinical problems vary in the degree to which they relate to a general factor of psychopathology (Lahey et al., 2012; Tackett, Lahey, et al., 2013). In the present study, this was largely replicated. Some forms of psychopathology (e.g. withdrawn depression) correlated strongly with the general psychopathology factor, while others were more representative of their specific factors. As the psychopathology and neuroticism models were largely overlapping, these discriminant correlations may be interpreted to reflect the extent to which neuroticism and its facets are core dispositional components of each disorder. The highly comorbid problems (e.g. withdrawn depression) seem to be characterized by a core of broad-spectrum negative affectivity, a finding in line with years of research on these disorders (Barlow et al., 2014; Kotov et al., 2010; Lahey et al., 2012; Tackett, Lahey, et al., 2013; Watson, 2005). However, as other disorders (e.g. conduct disorder, separation anxiety, and social phobia) were relatively independent of the general factor, this broad negative emotionality seems not to characterize them well. Instead, these same disorders tended to load heavily on their specific factors, and therefore may be better characterized as having a core of irritable or fearful negative affectivity. This corroborates prior studies of the general psychopathology bifactor, in which heavily fearbased disorders (e.g. phobias; Olino et al., 2014) are relatively independent of the $p$ factor in childhood. This is also in line with work suggesting that conduct disorder in children is related to 
callous-unemotionality, a core feature of which is a lack of emotional expressiveness (aside from anger/aggression; Frick \& Ellis, 1999; Kimonis et al., 2008). These results suggest that further research should be done to examine how dispositional traits, including others not analyzed here (e.g. positive emotionality) may factor into the core dispositional features of each form of psychopathology.

These findings underscore the importance of integrating hierarchical models of personality and psychopathology — this being an explicit goal of the HiTOP system (Kotov et al., 2017). Thus far, integration of personality and clinical domains has been done nearly exclusively at the level of Five Factor Model traits and psychopathology spectra (e.g., broad internalizing, thought disorder), despite that both traits and spectra represent single levels of more complex hierarchies. To date, relatively few studies have examined the relationship between traits and alternative levels of psychopathology structure, such as the general factor of psychopathology (Caspi et al., 2014; Olino et al., 2014; Tackett, Lahey, et al., 2013). As such, this study provides necessary insight into the connection between $p$ and another meaningful, dispositional latent variable - trait negative emotionality. Further, this present study demonstrates the utility of incorporating alternative levels of personality structure — facets, specifically —in differentiating the psychological content underlying spectra, as we found that specific facets of negative emotionality were discriminantly associated with specific variance in psychopathology spectra. Taken with other research in this area, these data support the conclusion that there is a need for a more extensive incorporation of psychopathology and personality measurement, as individual differences of multiple levels of dimensionality (including both higher-order traits and lowerorder facets) may play important, yet unique roles in the etiology and/or manifestation of internalizing and externalizing psychopathology. 


\section{Limitations \& Constraints on Generalizability}

The present study is limited by the need to rely on parents as sole informants for information about child personality and psychopathology (Soto, John, Gosling, \& Potter, 2008; Tackett, 2011), and that their reports were our only mode of assessment. As the nature of internalized negative emotions is, by definition, not easily observable, it is likely that parents cannot detect all true variance in internalized negative affectivity in either psychopathology and personality measures (Achenbach et al., 1987; De Los Reyes \& Kazdin, 2005). Further, it is possible that our observed correlations between parent-reported personality and psychopathology may be vulnerable to inflation through common response biases - a problem identified with many forms of single-informant research (Tackett, 2011; Wagner, Rau, \& Lindemann, 2010). Currently, however, there is no panacea to these measurement problems in either the child personality or psychopathology literature. In this study, child-reported psychopathology data was available, but as we already incorporated multiple independently developed assessments alongside multiple trait domains, models including child and parent report were beyond the scope of this paper. Beyond this, there are also substantial limitations on the validity and reliability of child self-reported personality and psychopathology data (De Los Reyes \& Kazdin, 2005; Soto et al., 2008). Future research examining these models with multiple informants and methods may help delineate the structure of these relationships and clarify method- and informant-specific boundaries around these research questions.

This study was conducted on a large, pooled sample of racially/ethnically diverse youth (with gender parity), and thus, our findings can be considered as a reasonable initial attempt at interrogating boundaries around representativeness of these findings across multiple racial/ethnic groups and genders. However, there are limitations to the extent to which these conclusions may 
be generalized beyond the population under present study. This research was conducted with community volunteer samples from urban environments in two developed countries in North America (Canada and the United States), and we primarily assessed common forms of psychopathology. The authors do not claim that these results should be generalized to populations aside from the one described (e.g., clinically severe samples or youths in developing countries) without further testing. Further, as thought disorder was not included in our psychopathology model due to a lack of sufficient indicators, these results should be interpreted with reference to commonalities in internalizing and externalizing problems. We might predict, based on prior research (e.g., (Caspi et al., 2014; Wright, Skodol, Hopwood, \& Morey, 2016), that a general factor model that includes thought disorder, personality disorders, and other omitted psychopathology indicators would yield similar results in this age group, however, this hypothesis requires testing in the future.

\section{Conclusions}

In this study, we found further support for the general factor model of psychopathology, as this model was found to be robust and replicable in a large, diverse sample of youth. We also found--for the first time--that the bifactor model psychopathology was highly overlapping with a bifactor model of neuroticism. These data suggested that common, or general, trait negative affectivity may account for much of the overlap between common psychiatric problems (i.e., the $p$ factor). We also found that the bifactor model of psychopathology allows us to better separate those aspects of psychopathology overlapping with general vulnerability factors (i.e. those overlapping with trait negative emotionality) and with specific vulnerability factors (i.e., those overlapping with specific fearfulness or irritability traits), relative to other dominant models. In short, the general psychopathology factor captures much of the same psychological content as 
general trait neuroticism/negative affectivity (i.e., the $n$ factor). Further, the Externalizing specific factor in this model is strongly correlated with specific variance in trait Irritability, while the Internalizing specific factor may also have a high correlation with specific variance in trait Fearfulness, though the magnitude of the latter relationship requires further testing. These data support the conclusion that neuroticism and its facets are pervasive at multiple levels of the hierarchical structure of psychopathology, pointing to a need for a more comprehensive integration of hierarchical models of personality and psychopathology in the future. 


\section{Author Contributions}

J.L.T. and C.M.B. developed the concept for the data analytic project reported here. The studies themselves were conceived of and designed by J.L.T., and data collection was performed by J.L.T., K.H., and A.J.S. C.M.B., J.L.T., and K.H. performed the data analysis. C.M.B. and A.J.S. drafted the paper, and K.H., A.J.S., and J.L.T. provided critical revisions. All authors approved the final version of the paper for submission.

\section{Acknowledgements}

The authors would like to thank the families who participated in the Child Personality and Behavioral Outcomes Study in both Toronto and Houston. We would also like to thank the members of the Personality Across Development Lab for their assistance with data collection. This research was supported in part by a grant from the Social Sciences and Humanities Research Council of Canada, awarded to J.L.T. 


\section{References}

Achenbach, T. M. (1966). The classification of children's psychiatric symptoms: A factoranalytic study. Psychological Monographs: General and Applied, 80(7), 1-37. http://dx.doi.org/10.1037/h0093906

Achenbach, T. M., \& Edelbrock, C. S. (1978). The classification of child psychopathology: a review and analysis of empirical efforts. Psychological Bulletin, 85(6), 1275.

Achenbach, T. M., McConaughy, S. H., \& Howell, C. T. (1987). Child/adolescent behavioral and emotional problems: Implications of cross-informant correlations for situational specificity. Psychological Bulletin, 101(2), 213-232.

Barlow, D. H., Sauer-Zavala, S., Carl, J. R., Bullis, J. R., \& Ellard, K. K. (2014). The Nature, Diagnosis, and Treatment of Neuroticism: Back to the Future . Clinical Psychological Science, 2(October 2013), 344-365. https://doi.org/10.1177/2167702613505532

Browne, M. W., \& Cudeck, R. (1992). Alternative ways of assessing model fit. Sociological Methods and Research, 21(2), 230-258.

Caspi, A., Houts, R. M., Belsky, D. W., Goldman-Mellor, S. J., Harrington, H., Israel, S., ... Moffitt, T. E. (2014). The p factor: One general psychopathology factor in the structure of psychiatric disorders? Clinical Psychological Science, 2(2), 119-137. https://doi.org/10.1177/2167702613497473

Clark, L. A., \& Watson, D. (1995). Constructing validity: Basic issues in objective scale development. Psychological Assessment, 7(3), 309-319.

Cronbach, L. J., \& Meehl, P. E. (1955). Construct Validity in Psychological Tests. Psychological Bulletin, 52(4), 22. 
De Bolle, M., Beyers, W., De Clercq, B., \& De Fruyt, F. (2012). General personality and psychopathology in referred and nonreferred children and adolescents: An investigation of continuity, pathoplasty, and complication models. Journal of Abnormal Psychology, 121(4), 958-970. https://doi.org/10.1037/a0027742

De Los Reyes, A., \& Kazdin, A. E. (2005). Informant Discrepancies in the Assessment of Childhood Psychopathology: A Critical Review, Theoretical Framework, and Recommendations for Further Study. Psychological Bulletin, 131(4), 483-509. https://doi.org/10.1037/0033-2909.131.4.483

De Pauw, S. S. W. (2017). Childhood Personality and Temperament. In T. A. Widiger (Ed.), The Oxford Handbook of the Five Factor Model (pp. 243-280). New York: Oxford University Press.

De Pauw, S. S. W., Mervielde, I., \& Van Leeuwen, K. G. (2009). How are traits related to problem behavior in Preschoolers? Similarities and contrasts between temperament and personality. Journal of Abnormal Child Psychology, 37(3), 309-325. https://doi.org/10.1007/s10802-008-9290-0

Eisenberg, N., Valiente, C., Spinrad, T. L., Cumberland, A., Reiser, M., Zhou, Q., \& Losoya, S. H. (2009). Longitudinal relations of children's effortful control, impulsivity, and negative emotionality to their externalizing, internalizing, and co-occurring behavior problems. Developmental Psychology, 45(4), 988-1008. https://doi.org/10.1037/a0016213.Longitudinal

Forbes, M. K., Tackett, J. L., Markon, K. E., \& Krueger, R. F. (2016). Beyond comorbidity: Toward a dimensional and hierarchical approach to understanding psychopathology 
across the life span. Development and Psychopathology, 28(4pt1), 971-986.

https://doi.org/10.1017/S0954579416000651

Frick, P. J., \& Ellis, M. (1999). Callous-unemotional traits and subtypes of conduct disorder. Clinical Child and Family Psychology Review, 2(3), 149-168.

Gjone, H., \& Stevenson, J. (1997). A longitudinal twin study of temperament and behavior problems: common genetic or environmental influences? Journal of the American Academy of Child \& Adolescent Psychiatry, 36(10), 1448-1456.

Griffith, J. W., Zinbarg, R. E., Craske, M. G., Mineka, S., Rose, R. D., Waters, A. M., \& Sutton, J. M. (2010). Neuroticism as a common dimension in the internalizing disorders. Psychological Medicine, 40(07), 1125-1136. https://doi.org/10.1017/S0033291709991449

Halverson, C. F., Havill, V. L., Deal, J. E., Baker, S. R., Victor, J. B., Pavlopoulos, V., ... Wen, L. (2003). Personality Structure as Derived from Parental Ratings of Free Descriptions of Children: The Inventory of Child Individual Differences. Journal of Personality, 71(6), 995-1026. https://doi.org/10.1111/1467-6494.7106005

Hettema, J. M., Neale, M. C., Myers, J. M., Prescott, C. A., \& Kendler, K. S. (2006). A population-based twin study of the relationship between neuroticism and internalizing disorders. American Journal of Psychiatry, 163(5), 857-864.

Hollingshead, A. B. (1975). Four Factor Index of Social Status. Unpublished Manuscript, New Haven, CT: Yale University.

Kim, H., \& Eaton, N. R. (2015). The hierarchical structure of common mental disorders: Connecting multiple levels of comorbidity, bifactor models, and predictive validity. 
Journal of Abnormal Psychology, 124(4), 1064-1078.

https://doi.org/10.1037/abn0000113

Kimonis, E. R., Frick, P. J., Skeem, J. L., Marsee, M. A., Cruise, K., Munoz, L. C., ... Morris, A. S. (2008). Assessing callous-unemotional traits in adolescent offenders: Validation of the Inventory of Callous-Unemotional Traits. International Journal of Law and Psychiatry, 31(3), 241-252. https://doi.org/10.1016/j.ijlp.2008.04.002

Kotov, R., Gamez, W., Schmidt, F., \& Watson, D. (2010). Linking “big” personality traits to anxiety, depressive, and substance use disorders: a meta-analysis. Psychol Bull, 136(5), 768-821. https://doi.org/10.1037/a0020327

Kotov, R., Krueger, R. F., Watson, D., Achenbach, T. M., Althoff, R. R., Bagby, R. M., ... Zimmerman, M. (2017). The Hierarchical Taxonomy of Psychopathology (HiTOP): A dimensional alternative to traditional nosologies. Journal of Abnormal Psychology, 126(4), 454-477. https://doi.org/10.1037/abn0000258

Krueger, R. F., Hicks, B. M., Patrick, C. J., Carlson, S. R., Iacono, W. G., \& McGue, M. (2002). Etiologic connections among substance dependence, antisocial behavior and personality: Modeling the externalizing spectrum. Journal of Abnormal Psychology, 111(3), 411-424. https://doi.org/10.1037/0021-843X.111.3.411

Krueger, R. F., \& Markon, K. E. (2006). Reinterpreting Comorbidity: A Model-Based Approach to Understanding and Classifying Psychopathology. Annual Review of Clinical Psychology, 2(1), 111-133. https://doi.org/10.1146/annurev.clinpsy.2.022305.095213

Krueger, R. F., \& Tackett, J. L. (2003). Personality and psychopathology: Working toward the bigger picture. Journal of Personality Disorders, 17(2: Special issue), 109-128. 
Kushner, S. C., Tackett, J. L., \& Bagby, R. M. (2012). The structure of internalizing disorders in middle childhood and evidence for personality correlates. Journal of Psychopathology and Behavioral Assessment, 34(1), 22-34. https://doi.org/10.1007/s10862-011-9263-4

Laceulle, O. M., Vollebergh, W. a. M., \& Ormel, J. (2015). The Structure of Psychopathology in Adolescence: Replication of a General Psychopathology Factor in the TRAILS Study. Clinical Psychological Science, (February), 1-11. https://doi.org/10.1177/2167702614560750

Lahey, B. B., Applegate, B., Chronis, A. M., Jones, H. A., Williams, S. H., Loney, J., \& Waldman, I. D. (2008). Psychometric Characteristics of a Measure of Emotional Dispositions Developed to Test a Developmental Propensity Model of Conduct Disorder. Journal of Clinical Child \& Adolescent Psychology, 37(4), 794-807. https://doi.org/10.1080/15374410802359635

Lahey, B. B., Applegate, B., Hakes, J. K., Zald, D. H., Hariri, A. R., \& Rathouz, P. J. (2012). Is there a general factor of prevalent psychopathology during adulthood? Journal of Abnormal Psychology, 121(4), 971-977. https://doi.org/10.1037/a0028355

Markon, K. E., Chmielewski, M., \& Miller, C. J. (2011). The reliability and validity of discrete and continuous measures of psychopathology: A quantitative review. Psychological Bulletin, 137(5), 856-879. https://doi.org/10.1037/a0023678

Martel, M. M., Pan, P. M., Hoffmann, M. S., Gadelha, A., do Rosário, M. C., Mari, J. J., ... Salum, G. A. (2016). A General Psychopathology Factor (P Factor) in Children: Structural Model Analysis and External Validation Through Familial Risk and Child Global Executive Function. Journal of Abnormal Psychology, (October). https://doi.org/10.1037/abn0000205 
Morgan, G., Hodge, K., Wells, K., \& Watkins, M. (2015). Are Fit Indices Biased in Favor of BiFactor Models in Cognitive Ability Research?: A Comparison of Fit in Correlated Factors, Higher-Order, and Bi-Factor Models via Monte Carlo Simulations. Journal of Intelligence, 3(1), 2-20. https://doi.org/10.3390/jintelligence3010002

Murray, A. L., Eisner, M., \& Ribeaud, D. (2016). The Development of the General Factor of Psychopathology 'p Factor' Through Childhood and Adolescence. Journal of Abnormal Child Psychology, 44(8), 1573-1586. https://doi.org/10.1007/s10802-016-0132-1

Nicholls, J. G., Licht, B. G., \& Pearl, R. A. (1982). Some Dangers of Using Personality Questionnaires To Study Personality. Psychological Bulletin, 92(3), 572-580.

Olino, T. M., Dougherty, L. R., Bufferd, S. J., Carlson, G. A., \& Klein, D. N. (2014). Testing models of psychopathology in preschool-aged children using a structured interview-based assessment. Journal of Abnormal Child Psychology, 42(7), 1201-1211. https://doi.org/10.1007/s10802-014-9865-X

Ormel, J., Bastiaansen, A., Riese, H., Bos, E. H., Servaas, M., Ellenbogen, M., ... Aleman, A. (2013). The biological and psychological basis of neuroticism: Current status and future directions. Neuroscience \& Biobehavioral Reviews, 37(1), 59-72. https://doi.org/10.1016/j.neubiorev.2012.09.004

Ormel, J., Jeronimus, B. F., Kotov, R., Riese, H., Bos, E. H., Hankin, B., ... Oldehinkel, A. J. (2013). Neuroticism and common mental disorders: Meaning and utility of a complex relationship. Clinical Psychology Review, 33(5), 686-697. https://doi.org/10.1016/j.cpr.2013.04.003 
R Core Team. (2017). R: A language and environment for statistical computing [R]. Vienna, Austria: R Foundation for Statistical Computing. Retrieved from https://www.Rproject.org/

Revelle, W. (2017). psych: Procedures for personality and psychology research (Version 1.7.8) [R]. Evanston, Illinois, USA: Northwestern University. Retrieved from https://CRAN.Rproject.org/package $=$ psych

Rosseel, Y. (2012). lavaan: An R package for structural equation modeling. Retrieved from http://www.jstatsoft.org/v48/i02/

Schmitz, S., Fulker, D. W., Plomin, R., Zahn-Waxler, C., Emde, R. N., \& DeFries, J. C. (1999). Temperament and problem behaviour during early childhood. International Journal of Behavioral Development, 23(2), 333-355.

semTools Contributors. (2016). semTools: Useful tools for structural equation modeling (Version 0.4-14) [R].

Shiner, R., \& Caspi, A. (2003). Personality differences in childhood and adolescence: Measurement, development, and consequences. Journal of Child Psychology and Psychiatry, 44(1), 2-32.

Simonds, J., \& Rothbart, M. K. (2004). The Temperament in Middle Childhood Questionnaire (TMCQ): A computerized self-report instrument for ages 7-10.

Snyder, H. R., Young, J. F., \& Hankin, B. L. (2017). Chronic Stress Exposure and Generation Are Related to the P-Factor and Externalizing Specific Psychopathology in Youth. Journal of Clinical Child \& Adolescent Psychology, 1-10. https://doi.org/10.1080/15374416.2017.1321002 
Soto, C. J., John, O. P., Gosling, S. D., \& Potter, J. (2008). The developmental psychometrics of big five self-reports: Acquiescence, factor structure, coherence, and differentiation from ages 10 to 20. Journal of Personality and Social Psychology, 94(4), 718-737. https://doi.org/10.1037/0022-3514.94.4.718

Soto, C. J., \& Tackett, J. L. (2015). Personality Traits in Childhood and Adolescence: Structure, Development, and Outcomes. Current Directions in Psychological Science, 24(5), 358362. https://doi.org/10.1177/0963721415589345

Suzuki, T., Samuel, D. B., Pahlen, S., \& Krueger, R. F. (2015). DSM-5 alternative personality disorder model traits as maladaptive extreme variants of the five-factor model: An itemresponse theory analysis. Journal of Abnormal Psychology, 124(2), 343-354. https://doi.org/10.1037/abn0000035

Tackett, J. L. (2006). Evaluating models of the personality-psychopathology relationship in children and adolescents. Clinical Psychology Review, 26(5), 584-599. https://doi.org/10.1016/j.cpr.2006.04.003

Tackett, J. L. (2011). Parent informants for child personality: Agreement, discrepancies, and clinical utility. Journal of Personality Assessment, 93(6), 539-544. https://doi.org/10.1080/00223891.2011.608763

Tackett, J. L., Herzhoff, K., Reardon, K. W., De Clercq, B., \& Sharp, C. (2014). The externalizing spectrum in youth: Incorporating personality pathology. Journal of Adolescence, 37(5), 659-668. https://doi.org/10.1016/j.adolescence.2013.10.009

Tackett, J. L., Herzhoff, K., Smack, A. J., Reardon, K. W., \& Adam, E. K. (2017). Does socioeconomic status mediate racial differences in the cortisol response in middle childhood? Health Psychology, 36(7), 662-672. https://doi.org/10.1037/hea0000480 
Tackett, J. L., Kushner, S. C., De Fruyt, F., \& Mervielde, I. (2013). Delineating personality traits in childhood and adolescence: associations across measures, temperament, and behavioral problems. Assessment, 20(6), 738-751.

https://doi.org/10.1177/1073191113509686

Tackett, J. L., \& Lahey, B. B. (2017). Neuroticism. In T. A. Widiger (Ed.), The Oxford Handbook of the Five Factor Model (pp. 39-56). New York, NY: Oxford University Press.

Tackett, J. L., Lahey, B. B., van Hulle, C., Waldman, I., Krueger, R. F., \& Rathouz, P. J. (2013). Common genetic influences on negative emotionality and a general psychopathology factor in childhood and adolescence. Journal of Abnormal Psychology, 122(4), 11421153. https://doi.org/10.1037/a0034151

Tackett, J. L., Slobodskaya, H. R., Mar, R. A., Deal, J., Halverson, C. F., Baker, S. R., ... Besevegis, E. (2012). The Hierarchical Structure of Childhood Personality in Five Countries: Continuity From Early Childhood to Early Adolescence. Journal of Personality, 80(4), 847-879. https://doi.org/10.1111/j.1467-6494.2011.00748.x

Wagner, S. M., Rau, C., \& Lindemann, E. (2010). Multiple Informant Methodology: A Critical Review and Recommendations. Sociological Methods \& Research, 38(4), 582-618. https://doi.org/10.1177/0049124110366231

Walton, K. E., Pantoja, G., \& McDermut, W. (2018). Associations Between Lower Order Facets of Personality and Dimensions of Mental Disorder. Journal of Psychopathology and Behavioral Assessment, 40(3), 465-475. https://doi.org/10.1007/s10862-017-9633-7

Walton, K. E., Roberts, B. W., Krueger, R. F., Blonigen, D. M., \& Hicks, B. M. (2008). Capturing Abnormal Personality With Normal Personality Inventories: An Item 
Response Theory Approach: Normal and Abnormal Personality Measures. Journal of Personality, 76(6), 1623-1648. https://doi.org/10.1111/j.1467-6494.2008.00533.x

Watson, D. (2005). Rethinking the mood and anxiety disorders: A quantitative hierarchical model for DSM-V. Journal of Abnormal Psychology, 114(4), 522-536. https://doi.org/10.1037/0021-843X.114.4.522

Wright, A. G. C., Skodol, A. E., Hopwood, C. J., \& Morey, L. C. (2016). Longitudinal Validation of General and Specific Structural Features of Personality Pathology. Journal of Abnormal Psychology, 125(8), 1120-1134.

Zinbarg, R. E., Mineka, S., Bobova, L., Craske, M. G., Vrshek-Schallhorn, S., Griffith, J. W., ... Anand, D. (2016). Testing a hierarchical model of neuroticism and its cognitive facets: Latent structure and prospective prediction of first onsets of anxiety and unipolar mood disorders during 3 years in late adolescence. Clinical Psychological Science, 4(5), 805824. 
Table 1

Psychopathology Measurement Model Fit and Factor Loadings

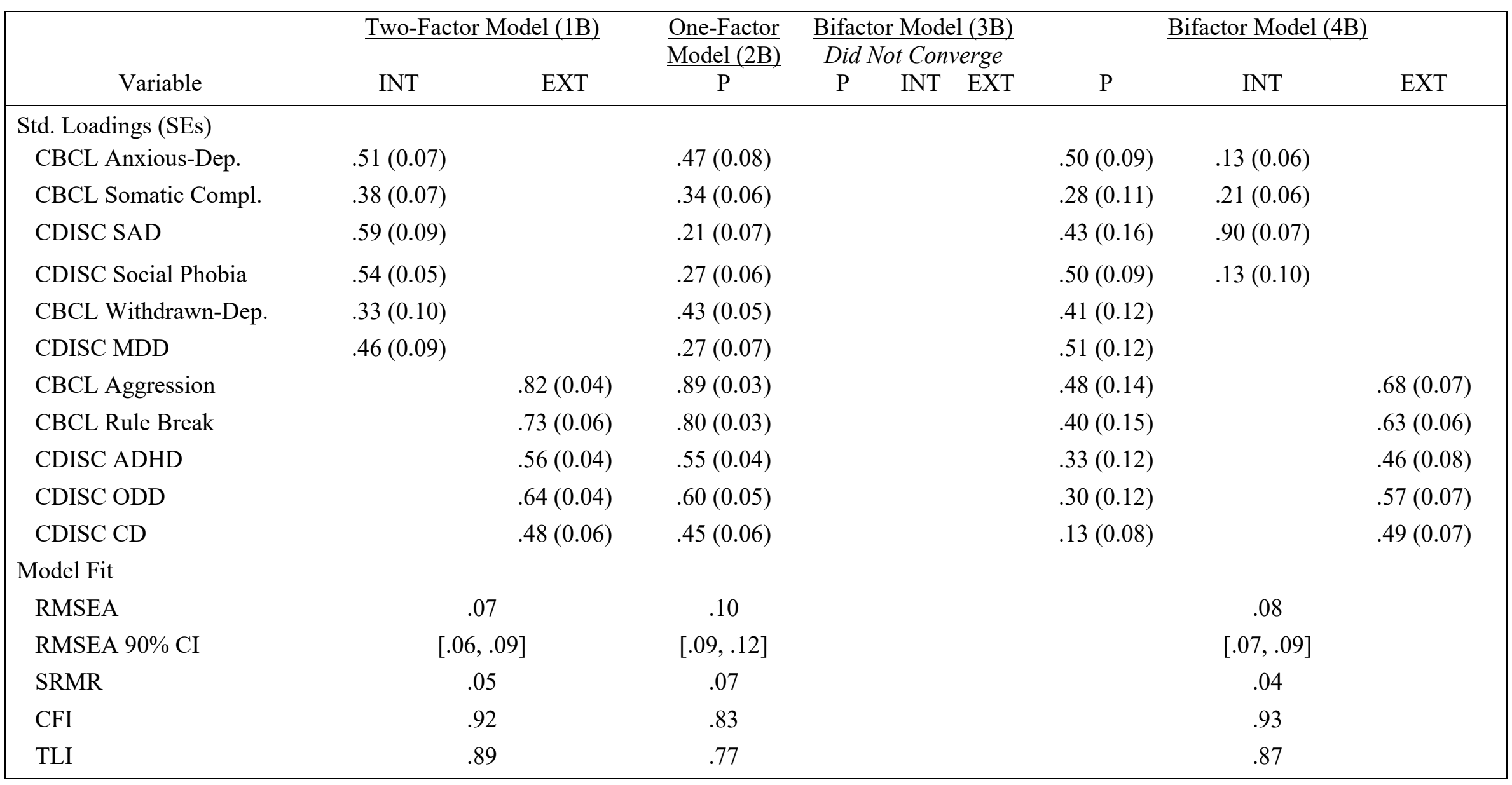


Table 2

Neuroticism Measurement Model Fit and Factor Loadings

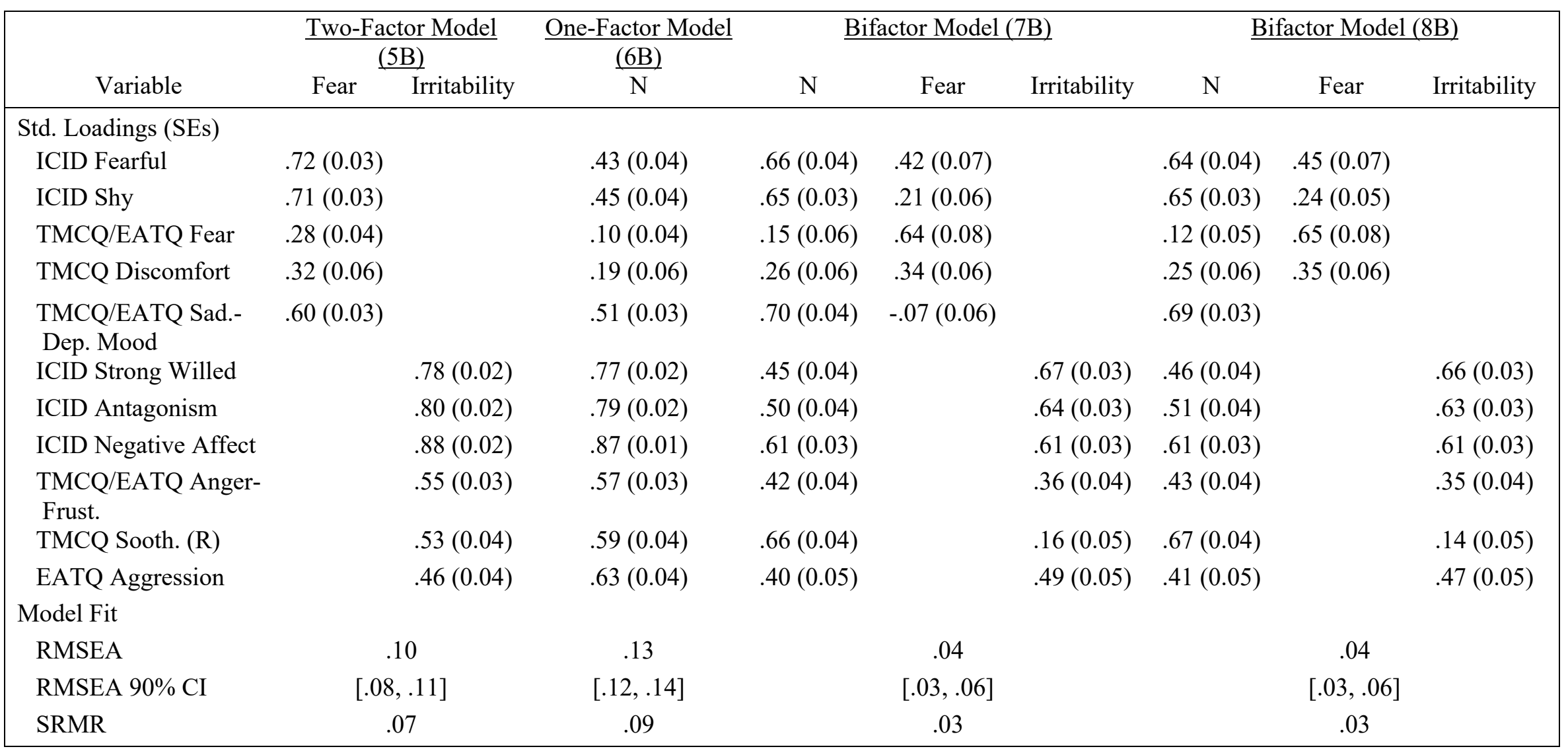


Table 3

Full Structural Model Fit, Factor Loadings, and Latent Variable Correlations

\begin{tabular}{|c|c|c|c|c|c|c|c|c|c|c|c|}
\hline \multirow[b]{3}{*}{ Variable } & \multicolumn{5}{|c|}{ Model 9B - Two Factor INT/EXT } & \multicolumn{6}{|c|}{ Model 10B - General Bifactor } \\
\hline & \multicolumn{2}{|c|}{ Psychopathology } & \multicolumn{3}{|c|}{ Neuroticism } & \multicolumn{3}{|c|}{ Psychopathology } & \multicolumn{3}{|c|}{$\underline{\text { Neuroticism }}$} \\
\hline & INT & EXT & $\mathrm{N}$ & Fear & Irritability & $\mathrm{P}$ & INT & EXT & $\mathrm{N}$ & Fear & Irritability \\
\hline \multicolumn{12}{|l|}{ Std. Loadings (SEs) } \\
\hline CBCL AnxDep & $.69(0.05)$ & & & & & $.68(0.06)$ & $.24(0.06)$ & & & & \\
\hline CBCL SomCom & $.41(0.06)$ & & & & & $.30(0.06)$ & $.32(0.06)$ & & & & \\
\hline CDISC SAD & $.45(0.08)$ & & & & & $.28(0.08)$ & $.55(0.09)$ & & & & \\
\hline CDISC Soc. Phob. & $.45(0.06)$ & & & & & $.36(0.06)$ & $.33(0.03)$ & & & & \\
\hline CBCL WithDep & $.55(0.06)$ & & & & & $.62(0.05)$ & & & & & \\
\hline CDISC MDD & $.40(0.07)$ & & & & & $.39(0.08)$ & & & & & \\
\hline CBCL Aggression & & $.87(0.03)$ & & & & $.56(0.06)$ & & $.68(0.04)$ & & & \\
\hline CBCL Rule Break. & & $.68(0.06)$ & & & & $.38(0.06)$ & & $.57(0.05)$ & & & \\
\hline CDISC ADHD & & $.54(0.04)$ & & & & $.34(0.06)$ & & $.42(0.05)$ & & & \\
\hline CDISC ODD & & $.67(0.04)$ & & & & $.38(0.05)$ & & $.57(0.04)$ & & & \\
\hline CDISC CD & & $.43(0.05)$ & & & & $.18(0.06)$ & & $.41(0.06)$ & & & \\
\hline ICID Fearful & & & $.64(0.07)$ & $.45(0.14)$ & & & & & $.68(0.04)$ & $.41(0.07)$ & \\
\hline ICID Shy & & & $.63(0.06)$ & $.23(0.12)$ & & & & & $.67(0.04)$ & $.13(0.06)$ & \\
\hline TMCQ/EATQ Fear & & & $.21(0.06)$ & $.63(0.19)$ & & & & & $.25(0.05)$ & $.61(0.06)$ & \\
\hline TMCQ Discomfort & & & $.33(0.08)$ & $.34(0.07)$ & & & & & $.33(0.08)$ & $.38(0.07)$ & \\
\hline $\begin{array}{l}\text { TMCQ/EATQ } \\
\text { Sad - Dep. Mood }\end{array}$ & & & $.74(0.06)$ & & & & & & $.72(0.03)$ & & \\
\hline ICID Strong Willed & & & $.49(0.04)$ & & $.62(0.04)$ & & & & $.49(0.04)$ & & $.63(0.04)$ \\
\hline ICID Antagonism & & & $.50(0.04)$ & & $.69(0.03)$ & & & & $.50(0.04)$ & & $.68(0.03)$ \\
\hline ICID Neg. Affect & & & $.64(0.04)$ & & $.57(0.04)$ & & & & $.64(0.03)$ & & $.58(0.03)$ \\
\hline TMCQ/EATQ Ang. & & & $.53(0.08)$ & & $.31(0.06)$ & & & & $.50(0.04)$ & & $.34(0.04)$ \\
\hline TMCQ Sooth.(R) & & & $.73(0.06)$ & & $.13(0.06)$ & & & & $.71(0.04)$ & & $.17(0.05)$ \\
\hline EATQ Aggression & & & $.46(0.08)$ & & $.49(0.06)$ & & & & $.44(0.05)$ & & $.52(0.04)$ \\
\hline \multicolumn{12}{|l|}{ Model Fit } \\
\hline RMSEA & & & .06 & & & & & & & & \\
\hline RMSEA 90\% CI & & & {$[.06, .07]$} & & & & & {$[.05$} & .06] & & \\
\hline SRMR & & & .06 & & & & & & & & \\
\hline \multicolumn{12}{|c|}{ Latent Variable Correlations } \\
\hline Internalizing & - & & & & & & & & & & \\
\hline Externalizing & .54 & - & & & & & & & & & \\
\hline $\mathrm{N}$ & .76 & .49 & - & & & .81 & - & - & & & \\
\hline
\end{tabular}




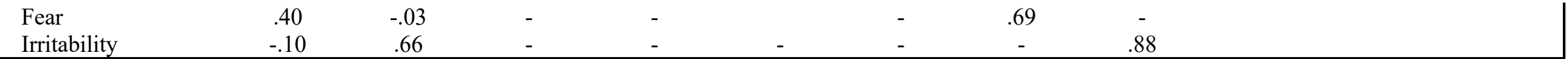

Table 4

Exploratory Latent Variable and Factor Score Correlations \& 95\% Confidence Intervals

\begin{tabular}{|lllllll|}
\hline & INT & $\mathrm{P}$ & EXT & Fear & $\mathrm{N}$ & Irrit \\
$\mathrm{INT}$ & 1 & - & - & $.54[.09, .99]$ & $-.38[-1.44, .68]$ & $.21[-.32, .73]$ \\
$\mathrm{P}$ & - & 1 & - & $.20[.01, .40]$ & $.99[.66,1.34]$ & $-.19[-.41, .03]$ \\
EXT & - & - & 1 & $-.21[-.38,-.03]$ & $-.11[-.36, .15]$ & $.94[.82,1.06]$ \\
Fear & $.22[.10, .33]$ & $.19[.08, .30]$ & $-.20[-.31,-.09]$ & 1 & - & - \\
$\mathrm{N}$ & $.00[-.08, .07]$ & $.49[.39, .57]$ & $.25[.14, .36]$ & - & 1 & - \\
Irritability & $-.08[-.18, .02]$ & $.22[.11, .33]$ & $.61[.53, .68]$ & - & - & 1 \\
\hline
\end{tabular}

Note: The upper diagonal shows correlations from the version of Model 10B that freed all psychopathology-neuroticism latent variable correlations, though it should be noted that this correlation matrix was not positive definite. The lower diagonal shows correlations between all factor scores. 
Figure 1

Full Bifactor Structural Model (10B)

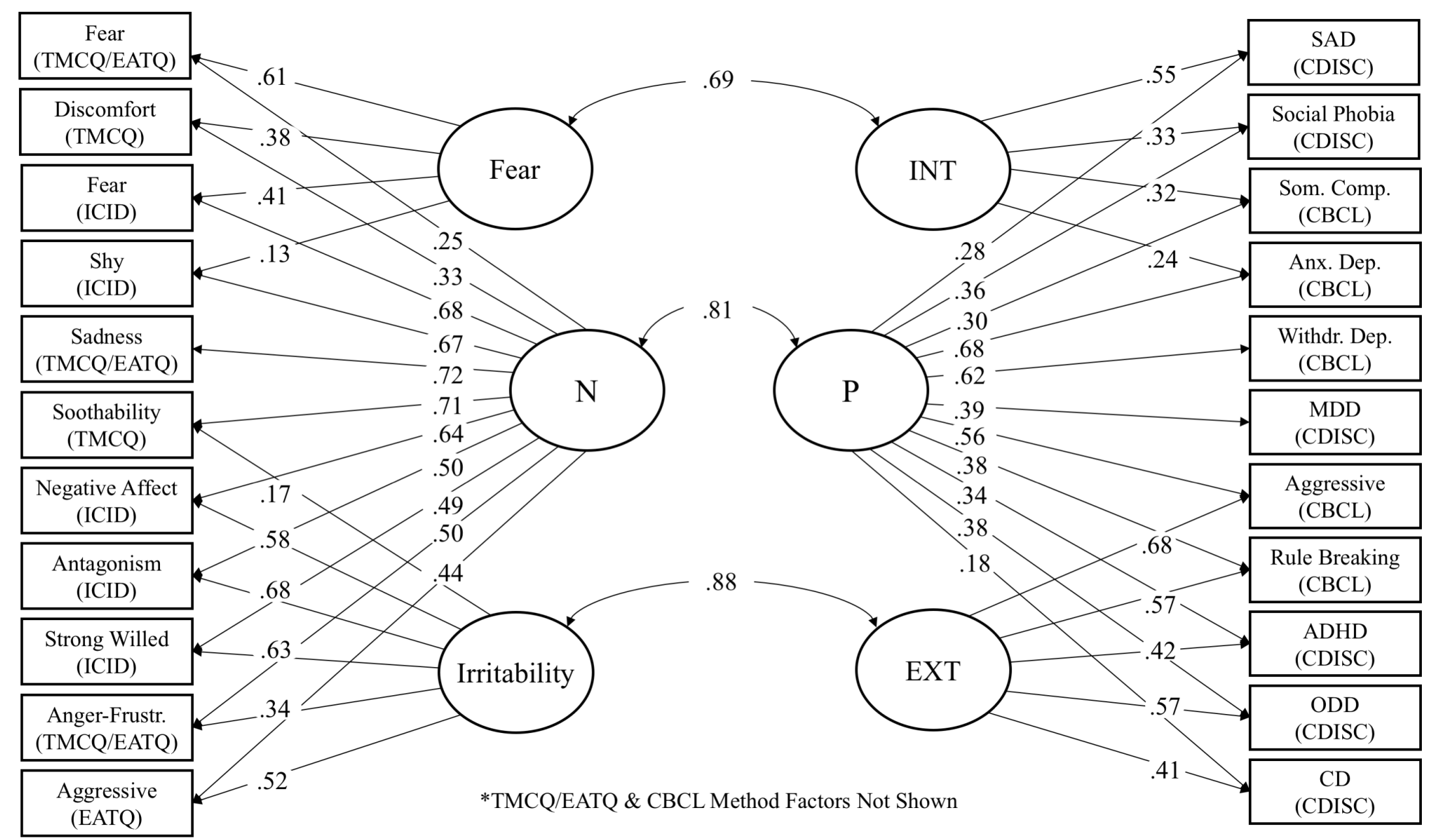

